\title{
Ovarian Ectopic Pregnancy Following Assisted Reproductive Techniques: A Rare Entity
}

\author{
${ }^{1}$ Harpreet Kaur, ${ }^{2} \mathrm{~T}$ Shashikala, ${ }^{2}$ Meenakshi Bharath, ${ }^{2}$ Nivedita Shetty, ${ }^{2}$ Kamini A Rao \\ ${ }^{1}$ Fellow in Reproductive Medicine, Reproductive Medicine Unit, Bangalore Assisted Conception Centre, Bengaluru, Karnataka, India \\ ${ }^{2}$ Consultant in Reproductive Medicine, Reproductive Medicine Unit, Bangalore Assisted Conception Centre, Bengaluru, Karnataka, India
}

Correspondence: Harpreet Kaur, Fellow in Reproductive Medicine, Reproductive Medicine Unit, Bangalore Assisted Conception Centre, Bengaluru-560001, Karnataka, India, Phone: 09632229118, 09872221818, e-mail: drharpreet_sidhu@ hotmail.com husanmeet@yahoo.co.in

\section{ABSTRACT}

Ovarian ectopic pregnancy should be kept in mind as a rare possibility following assisted reproductive techniques. Although early use of quantitative serum $\beta$-hCG and pelvic ultrasonography has increased our diagnostic capability for ectopic pregnancy, ovarian pregnancy still represents a diagnostic problem. Laparoscopy is the gold standard for diagnosis and treatment for ovarian ectopic pregnancy.

Keywords: Ovarian ectopic, Assisted reproductive techniques, Laparoscopy.

\section{INTRODUCTION}

Ovarian pregnancy is a rare variety of ectopic implantation. Its incidence after natural conception varies from one in 7000 to one in 16,000 delieveries and accounts for 1 to $3 \%$ of all ectopic gestations. ${ }^{1,2}$ Although the incidence of ectopic pregnancy is on rise after assisted reproductive technology, ovarian pregnancy is still a rare event. It occurs when an ovum is fertilized and retained within the ovary. ${ }^{1,3}$ Here, we are presenting two cases of ovarian ectopic pregnancy following assisted reproductive techniques.

\section{CASE REPORTS}

First case was of a 29-year-old with complaints of primary infertility for four years. She had regular cycles with normal hormonal profile. Her husband was detected to have oligoasthenospermia. During index pregnancy, she conceived following IVF, which was planned in view of abnormal semen parameters.

She was given long agonist protocol, down-regulation was achieved with Leupride acetate injection followed by ovarian stimulation with recombinant FSH injection. Eighteen MII oocytes were retrieved and three, eight cell, grade A embryos were transferred on day three following oocyte retrieval under ultrasonography guidance using Wallace catheter (Sure view ${ }^{\circledR}$ Wallace ${ }^{\circledR}$ embryo replacement catheter, Smith medical international Ltd. Brisbane, Australia).

Two weeks following embryo transfer, her serum $\beta$-hCG level was 25.0 IU/L. Repeat serum $\beta$-hCG one week later was

Date of Received: 14-08-10

Date of Acceptance: 18-08-10

Date of Publication: Jan. 2011
1415.0 IU/L and TVS showed no evidence of intrauterine/ extrauterine gestation sac, corpus luteum was seen on right ovary. Repeat serum $\beta$-hCG five days later was 6550.0 IU/L and transvaginal ultrasonography showed presence of right adnexal geastation sac measuring $67 \mathrm{~mm}$. Endometrium was eight mm thick with no evidence of intrauterine gestation sac. Minimal free fluid was present in pelvis. With the clinical diagnosis of right tubal ectopic pregnancy she was given injection. Methotrexate $50 \mathrm{mg}$ IM on 12 April 2010 at 6 pm after checking the hemogram, renal and liver function test. On 13 April 2010 at 7 am, she complained of severe abdominal pain. On examination, her pulse rate was $82 / \mathrm{min}$ and BP was 120/80 mm Hg with no obvious pallor and tenderness on abdominal examination. USG showed right adnexal gestation sac with evidence of moderate amount of hemoperitoneum. With the diagnosis of ruptured tubal pregnancy, she was taken up for operative laparoscopy. Intraoperatively, approximately $500 \mathrm{ml}$ of hemoperitoneum was drained. Uterus was normal and bilateral tubes were inspected meticulously and were found to be normal. Left ovary appeared normal. On right side, there was ovarian ectopic pregnancy measuring $2 \times 1.5 \mathrm{~cm}$ with active bleeding from surface and friable consistency (Fig. 1). The gestational tissue was resected from the right ovary and base was cauterized ensuring complete hemostasis. Endometrial curettings were sent for histopathological examination. Postoperative period was uneventful. Repeat serum $\beta$-hCG one week following surgery was $185.3 \mathrm{IU} / \mathrm{L}$ and it decreased to $<10$ IU/L within three weeks following surgery. Histopathology revealed chorionic villi with presence of large areas of hemorrhage and fibrocollagenous stroma suspicious of ovarian tissue. Tissue from endometrium revealed decidualized endometrial stroma with secretory endometrial glands (Fig. 2). 
Second case, 34-year-old primigravida presented at six weeks, gestation with ultrasonographic diagnosis of left ovarian ectopic pregnancy. She conceived following ovulation induction with letrozole and human menopausal gonadotropin and intrauterine insemination. Her first $\beta$-hCG level was $3810.0 \mathrm{IU} / \mathrm{L}$ on 10 June 2010, transvaginal scan done on the same day showed irregular sac-like structure in the uterine cavity and B/L adnexa was normal. Repeat $\beta$-hCG on 16 June 2010 was $12,481.00 \mathrm{IU} / \mathrm{L}$ and USG revealed left ovarian ectopic gestation sac with presence of fetal pole and fetal cardiac activity (Fig. 3). Her vitals were stable. She was planned for operative laparoscopy on 17 June 2010. Intraoperatively, uterus and both the fallopian tubes were found to be normal. On left side, unruptured ovarian ectopic was present, with congestion and bluish discoloration of left ovary (Fig. 4). Products of conception were removed completely and hemostasis ensured by cauterization. Postoperatively her $\beta$-hCG was normal in four weeks. Histopathology revealed trophoblastic tissue with chorionic villi in ovarian tissue and secretory, decidualized endometrium.

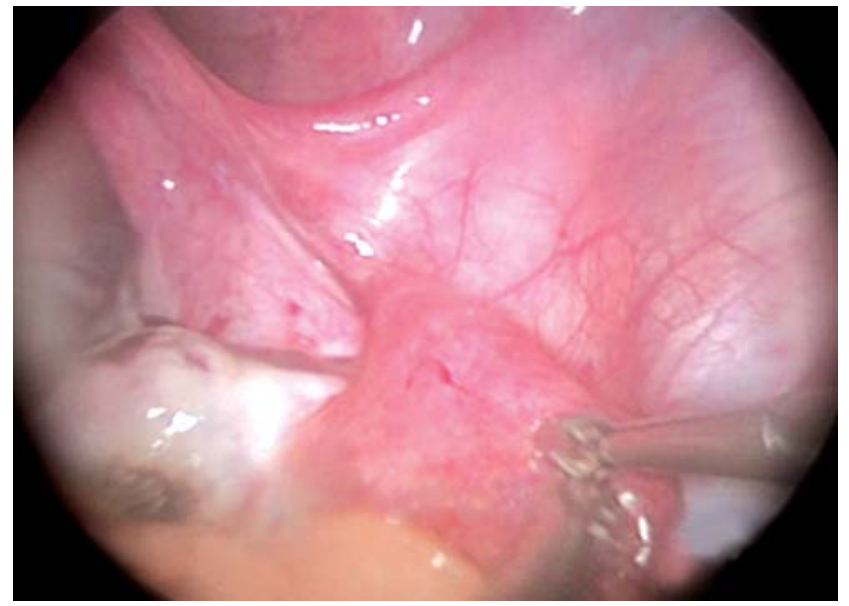

Fig. 1: Laparoscopic view of right ovarian ectopic pregnancy with normal looking fallopian tube

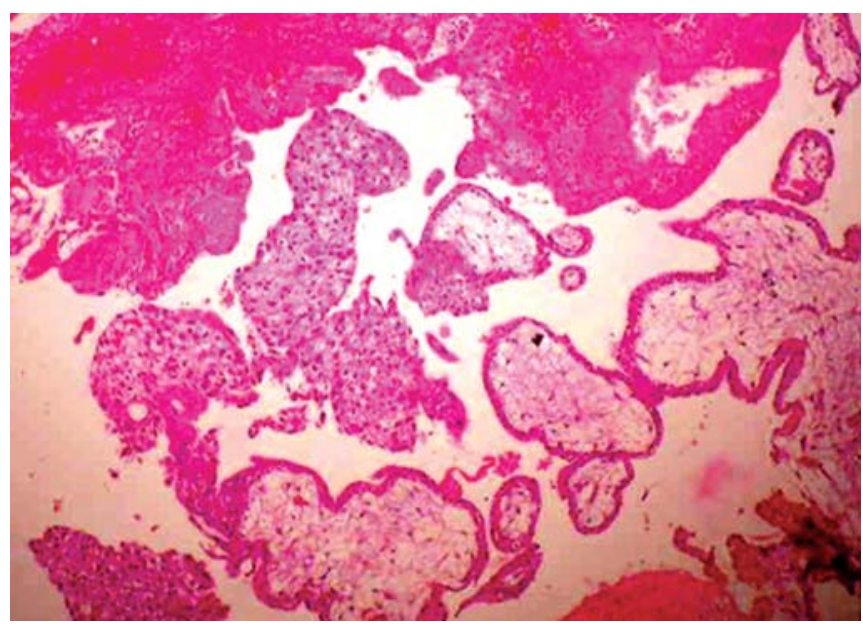

Fig. 2: Section from right ovarian biopsy showing chorionic villi with hemorrhage (Hematoxyline-Eosin stain; OM × 100)

\section{DISCUSSION}

Ovarian pregnancy is a rare form of ectopic gestation. The cause for development of an ovarian pregnancy is a result of secondary implantation or failure of follicular extrusion. This form of implantation could be result of reverse migration of embryos as a result of deep deposition of the embryos into the uterine cavity, ${ }^{4}$ or the use of large volume of culture fluid during transfer. ${ }^{5}$ The presence of tubal pathology and pelvic inflammatory disease could be predisposing factors as with tubal pregnancies. The distinction of primary or secondary ovarian pregnancy is no longer of practical significance. ${ }^{6}$ The widely quoted classification of primary ovarian pregnancy into intrafollicular or extrafollicular type is often not feasible. Moreover, such a classification has no therapeutic value. ${ }^{6}$ Rarely
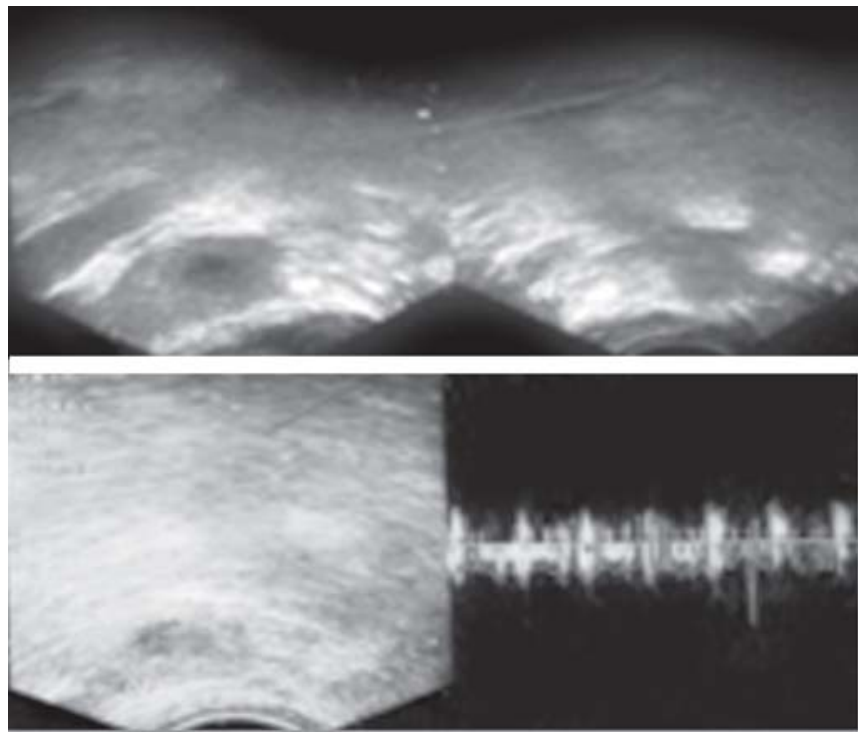

Fig. 3: Transvaginal ultrasonography showing left ovarian ectopic pregnancy with presence of fetal cardiac activity

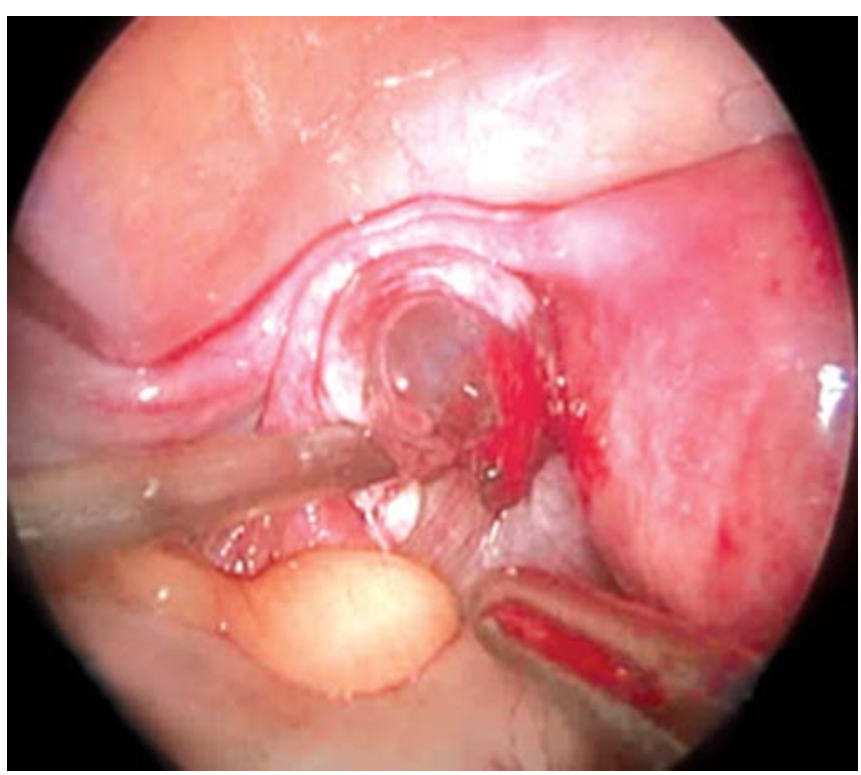

Fig. 4: Laparoscopic view of left ovarian ectopic pregnancy with normal looking fallopian tube 
ovarian pregnancy can be a part of heterotopic or of a twin ovarian pregnancy.

Ovarian pregnancy can occur without any classical antecedent risk factors; however, there seems to be strong association with intrauterine contraceptive device. Other risk factors are assisted reproductive technology, endometriosis or PID. The index cases-first resulted following IVF-ET and second following intrauterine insemination. Common clinical symptoms are abdominal pain with or without vaginal bleeding. Although early use of quantitative serum $\beta$-hCG and pelvic ultrasonography has increased our diagnostic capability for ectopic implantation, ovarian pregnancy still represents a diagnostic problem. ${ }^{1,6}$ A more echogenic white ring in the ovary compared with the ovarian tissue with a yolk sac or fetal parts are the key ultrasonography indicators of ovarian ectopic, however an embryo is relatively infrequently seen. ${ }^{3,7}$ Both sonographically and at the time of surgery, clinical challenge is to distinguish an ovarian ectopic pregnancy from corpus luteum or hemorrhagic cyst. ${ }^{1,7}$ Decreased wall echogenicity compared with the endometrium and anechoic texture suggests corpus luteum and diagnosis can be confirmed by histopathological examination after surgery. ${ }^{7,8}$ In the first case, ovarian pregnancy was diagnosed intraoperatively as there was active bleeding from the pregnancy implanted in the ovary and both the fallopian tubes were normal, while second case was diagnosed preoperatively as ovarian ectopic pregnancy. As suggested by Spigielberg (1878), primary ovarian pregnancy must be distinguished from distal tubal pregnancy, a condition that can secondarily involve the ovary at its surface. ${ }^{9}$ He outlined four criteria for the diagnosis of primary ovarian gestation:

1. The fallopian tube with its fimbriae should be intact and separate from the ovary.

2. The gestational sac should occupy the normal position of the ovary.

3. The gestational sac should be connected to uterus by the utero-ovarian ligament.

4. Ovarian tissue must be preserved in the specimen attached to the gestational sac. ${ }^{3,6,9}$ Though our cases failed to fulfill the last criteria, but any ovarian tissue present at base could have been destroyed following cauterization to achieve hemostasis. Due to coagulation artefacts, it may be difficult to find ovarian tissue around the gestational sac microscopically. ${ }^{6,10}$ So, a modification of fourth criteria of Spigelberg is the detection of chorionic villi without concurrent detection of intact ovarian parenchyma, is sufficient today for a selective laparoscopic biopsy. ${ }^{6,10,11}$

Laparoscopy is the mainstay of treatment for ovarian ectopic. Preferred method is ovarian wedge resection or cystectomy. Medical treatment has a limited role as diagnosis of ovarian ectopic may be difficult until we perform laparoscopy. So, if laparoscopy is required for diagnosis, definitive surgical management may be accomplished at the same time. Ultrasound-guided local injection of methotrexate or PGF2 $\alpha$ has been reported in limited number of case reports only. ${ }^{10,11}$ So, medical treatment is mainly indicated as a secondary option for primary incomplete resection or trophoblastic persistence. ${ }^{10,11}$ Unlike the tubal ectopic, which has a significant risk of recurrence, to date there has been no reports of repeat ovarian ectopic pregnancy. ${ }^{2}$ In our cases, laparoscopic resection of ovarian ectopic and thermal coagulation was done to achieve hemostasis. Serial serum $\beta$-hCG follow-up was done until it became negative. So, ovarian ectopic pregnancy should be kept in mind as a rare possibility following ART. Laparoscopy is the gold standard for diagnosis and treatment for ovarian ectopic pregnancy.

\section{REFERENCES}

1. Bontis J, Grimbizis G, Tarlastzis BC, Miliaras D, Billi H. Intrafollicular ovarian pregnancy following ovulation induction/ intrauterine insemination: Pathophysiological aspects and diagnostic problems. Hum Reprod 1997;12(2):376-78.

2. Nadarajah S, Sim LN, Loh SF. Laparoscopic management of ovarian pregnancy. Singapore Med J 2002;43(2):95-96.

3. Kraemer B, Kraemer E, Guengoer E, Juhasz-Boess I, Solomayer EF, Diethelm W, et al. Ovarian ectopic pregnancy: Diagnosis, treatment, correlation to Carnegie stage 16 and review based on a clinical case. Fertil and Steril 2009;92(1):392e13-15.

4. Pope CS, Cook EK, Arny M, Novak, Grow DR. Influence of embryo transfer depth on in vitro fertilization and embryo transfer outcome. Fertil and Steril 2004;81:51-58.

5. Zouves C, Erenus M, Gomel V. Tubal ectopic pregnancy after in vitro fertilization and embryo transfer: A role for proximal occlusion or salpingectomy after failed distal tubal surgery. Fertil Steril 1991.

6. Seinera P, Di Gregorio A, Arisio R, Decko A, Crana F. Ovarian pregnancy and operative laparoscopy: Report of eight cases. Hum Reprod 1997;12(3):608-10.

7. Stein MW, Ricci ZJ, Novak L, Roberts JH, Koenigsberg M. Sonographic comparasion of the tubal ring of ectopic pregnancy with the corpus luteum. J Ultrasound Med 2004;23:57-62.

8. Comstock C, Huston K, Lee W. The ultrasonographic appearance of ovarian ectopic pregnancies. Obstet Gynecol 2005;105(1):42-45.

9. Spiegelberg O. Zur kasuistik der ovarialschwangerschaft. Arch Gynaekol 1878;13:73-79.

10. Einenkel J, Baier D, Horn LC, Alexander H. Laparoscopic therapy of an intact primary ovarian pregnancy with ovarian hyperstimulation syndrome. Hum Reprod 2000;15(9):203740.

11. Chelmow D, Gates E, Penzias AS. Laparoscopic diagnosis and methotrexate treatment of an ovarian pregnancy: A case report. Fertil Steril 1994;62:879-81. 